\title{
Heating and atom loss during upward ramps of Feshbach resonance levels in Bose-Einstein condensates
}

\author{
Thorsten Köhler, ${ }^{1}$ Krzysztof Góral, ${ }^{1,2}$ and Thomas Gasenzer ${ }^{3}$ \\ ${ }^{1}$ Clarendon Laboratory, Department of Physics, University of Oxford, Parks Road, Oxford, OX1 3PU, United Kingdom \\ ${ }^{2}$ Center for Theoretical Physics, Polish Academy of Sciences, Aleja Lotników 32/46, 02-668 Warsaw, Poland \\ ${ }^{3}$ Institut für Theoretische Physik, Universität Heidelberg, Philosophenweg 16, 69120 Heidelberg, Germany
}

(Dated: October 29, 2018)

\begin{abstract}
The production of pairs of fast atoms leads to a pronounced loss of atoms during upward ramps of Feshbach resonance levels in dilute Bose-Einstein condensates. We provide comparative studies on the formation of these bursts of atoms containing the physical predictions of several theoretical approaches at different levels of approximation. We show that despite their very different description of the microscopic binary physics during the passage of a Feshbach resonance, all approaches lead to virtually the same prediction on the total loss of condensate atoms, provided that the ramp of the magnetic field strength is purely linear. We give the reasons for this remarkable insensitivity of the remnant condensate fraction to the microscopic physical processes and compare the theoretical predictions with recent Feshbach resonance crossing experiments on ${ }^{23} \mathrm{Na}$ and ${ }^{85} \mathrm{Rb}$.
\end{abstract}

PACS numbers: 03.75.Kk, 34.50.-s, 05.30.-d

\section{INTRODUCTION}

Since their first experimental demonstration [1], magnetic field tunable interactions have proven their potential and usefulness in the physics of quantum degenerate gases. This technique takes advantage of the Zeeman effect in the electronic energy levels of the atoms to manipulate their binary collision properties in the vicinity of a Feshbach resonance. For several atomic species it is now possible to change the scattering length by orders of magnitude, and even to reverse its sign, making the atoms either repel or attract one another. One of the impressive applications of this technique was the successful creation of stable ${ }^{85} \mathrm{Rb}$ Bose-Einstein condensates with thousands of atoms [2], that would otherwise collapse if consisting of more than about 80 atoms. These experiments have now been refined to associate pairs of atoms to ultracold molecules and probe their coherence properties [3, 4, 5, 6, 7, 8, 9, 10, 11, 12, 13, 14, 15, 16, 17, 18, 19, 20]. The dissociation energies of the highly excited dimer molecules have been measured accurately down to several $\mathrm{kHz}[3,4,5]$.

Early attempts to associate Feshbach molecules in BoseEinstein condensates [21] relied upon linear ramps of the magnetic field strength across the resonance position, in which the resonance level (see, e.g., [22, 23]) was swept upward in energy [24]. Subsequent theoretical studies [22, 25] have shown that these particular ramps lead to a heating of the cloud rather than to molecular production. The condensate atoms were lost into correlated pairs of atoms with a comparatively high velocity of their relative motion. These atom pairs were also produced in the interferometric experiments of Ref. [3], involving a special sequence of magnetic field pulses, and directly detected as a dilute burst of atoms with a spread far beyond the size of the remnant Bose-Einstein condensate. The theoretical studies in Refs. [26, 27, 28] have confirmed this interpretation and provided a quantitative understanding of the number of burst atoms observed in Ref. [3] as well as their measured energy spectrum [28].

Several approaches have been proposed to describe the con- version of condensate atoms into bound or continuum molecular states (see, e.g., [22, 23, 25, 26, 27, 28, 29, 30, 31, 32, 33, 34, 35]). Early approaches are based either on mean field theory, with an exchange of particles between the atomic condensate and the Feshbach resonance level (e.g. [25, 29, 30]), or on a two-body configuration interaction picture, extended in such a way that it includes the Bose stimulation of the transition between free atom pairs and the resonance level [22]. While these approaches effectively include only two energy levels of the atom pairs, i.e. the zero energy scattering state and the Feshbach resonance level [22, 23], the interferometric experiments in Ref. [3] have clearly revealed that a complete description of the molecular association requires many-body approaches, which treat the two-body collision dynamics including all states in a non-perturbative manner [26, 28, 32].

In this paper we use the beyond mean field approach of Refs. [28, 32] to describe the atom loss in upward ramps of a Feshbach resonance level in a Bose-Einstein condensate and compare the results with the simpler two level mean field approach of Refs. [25, 29, 30]. Our studies involve the Feshbach resonance crossing experiments of Refs. [21] and [2]. In the experiments [21] a Bose-Einstein condensate of ${ }^{23} \mathrm{Na}$ in the $\left(F=1, m_{F}=+1\right)$ hyperfine state was exposed to a magnetic field swept across the Feshbach resonances at 853 and 907 G [1], respectively. In Ref. [2] the magnetic field strength was swept across the $155 \mathrm{G}$ resonance of a gas of ${ }^{85} \mathrm{Rb}$ atoms, which was condensed in the $\left(F=2, m_{F}=-2\right)$ hyperfine state. In all cases dramatic losses of atoms were observed, which increased with decreasing ramp speed.

The widths of the ${ }^{23} \mathrm{Na}$ Feshbach resonances have been predicted to be $1 \mathrm{G}$ (at $907 \mathrm{G}$ ) and $0.01 \mathrm{G}$ (at $853 \mathrm{G}$ ), respectively [1]. The ${ }^{85} \mathrm{Rb} 155 \mathrm{G}$ Feshbach resonance has been characterised very accurately in several experimental and theoretical studies [3, 4, 36, 37], and its width is predicted to be $11 \mathrm{G}$. We shall show on the basis of binary collision physics that the microscopic dynamics caused by the ramps of the magnetic field strength is decisively different between the ${ }^{23} \mathrm{Na}$ experiments [21] and the experiments [2] with the broad ${ }^{85} \mathrm{Rb}$ Feshbach resonance, although the direction of these resonance crossings 
excludes the adiabatic association of atoms to molecules (see, e.g., [22, 23]) in both cases. We shall show that a two level picture is particularly inappropriate to describe the Feshbach resonance crossing in ${ }^{85} \mathrm{Rb}$ [2]. Our studies reveal, however, that both the first order microscopic quantum dynamics approach of Refs. [28, 32] and the two level mean field theory of Refs. [25, 29, 30] predict largely the same atom loss for the experiments in Refs. [21] and [2]. We show that this remarkable insensitivity of the remnant condensate fraction with respect to the description of the microscopic binary collision physics is characteristic for linear ramps of the magnetic field strength and provide the essential physical parameters that determine the losses. We show furthermore that our findings are quite general as they also apply to the dissociation spectra of Feshbach molecules in upward ramps of the Feshbach resonance level, which are widely used for the purpose of molecular de- tection (see, e.g., [6, 7, 15, 38]). We note, however, that the interferometric experiments of Refs. [3, 4] provide an example of a sequence of magnetic field pulses, which as a whole varies non-linearly in time. These experiments indeed reveal details of the microscopic binary collision physics beyond universal considerations [26, 28, 39].

\section{MICROSCOPIC QUANTUM DYNAMICS APPROACH}

In general, the interatomic interaction depends on the relative coordinates of the atoms and couples all their hyperfine energy levels, labelled in the following by Greek indices. A trapped gas of Bose atoms is then described by the many-body Hamiltonian:

$$
H=\sum_{\mu} \int d \mathbf{x} \psi_{\mu}^{\dagger}(\mathbf{x}) H_{\mu}^{1 \mathrm{~B}}(B) \psi_{\mu}(\mathbf{x})+\frac{1}{2} \sum_{\mu \nu \kappa \lambda} \int d \mathbf{x} \int d \mathbf{y} \psi_{\mu}^{\dagger}(\mathbf{x}) \psi_{\nu}^{\dagger}(\mathbf{y}) V_{\{\mu \nu\},\{\kappa \lambda\}}(\mathbf{x}-\mathbf{y}) \psi_{\kappa}(\mathbf{x}) \psi_{\lambda}(\mathbf{y})
$$

Here $H_{\mu}^{1 \mathrm{~B}}(B)=-\hbar^{2} \nabla^{2} / 2 m+V_{\mu}^{\mathrm{trap}}+E_{\mu}^{\mathrm{hf}}(B)$ is the Hamiltonian of a single atom in the hyperfine state $\mu$ containing the kinetic energy, the trap potential and the hyperfine energy that depends on the magnetic field strength $B$ due to the Zeeman effect, and $m$ is the atomic mass. $V_{\{\mu \nu\},\{\kappa \lambda\}}(\mathbf{r})$ is the potential associated with the asymptotic incoming and outgoing binary scattering channels $\{\kappa \lambda\}$ and $\{\mu \nu\}$, which depends on the relative coordinates $\mathbf{r}=\mathbf{x}-\mathbf{y}$. The field operators obey the Bose commutation relations $\left[\psi_{\mu}(\mathbf{x}), \psi_{\nu}^{\dagger}(\mathbf{y})\right]=\delta_{\mu \nu} \delta(\mathbf{x}-\mathbf{y})$ and $\left[\psi_{\mu}(\mathbf{x}), \psi_{v}(\mathbf{y})\right]=0$.

All physical quantities of a gas are determined by correlation functions, i.e., expectation values (denoted by $\langle\cdots\rangle)$ of normal ordered products of field operators in the state at time $t$. Quantities of particular interest are the mean field $\Psi_{\mu}(\mathbf{x}, t)=\left\langle\psi_{\mu}(\mathbf{x})\right\rangle_{t}$, the pair function $\Phi_{\mu \nu}(\mathbf{x}, \mathbf{y}, t)=\left\langle\psi_{v}(\mathbf{y}) \psi_{\mu}(\mathbf{x})\right\rangle_{t}-\Psi_{v}(\mathbf{y}, t) \Psi_{\mu}(\mathbf{x}, t)$ and the one-body density matrix of the non-condensed fraction $\Gamma_{\mu \nu}(\mathbf{x}, \mathbf{y}, t)=$ $\left\langle\psi_{\gamma}^{\dagger}(\mathbf{y}) \psi_{\mu}(\mathbf{x})\right\rangle_{t}-\Psi_{v}^{*}(\mathbf{y}, t) \Psi_{\mu}(\mathbf{x}, t)$. We have shown in Refs. [28, 32] how the exact infinite hierarchy of dynamic equations for correlation functions can be truncated to any desired degree of accuracy. In the first order approach [28] the mean field and the pair function are determined by the coupled dynamic equations [23]:

$$
\begin{aligned}
i \hbar \frac{\partial}{\partial t} \Psi_{\mu}(\mathbf{x}, t) & =H_{\mu}^{1 \mathrm{~B}}(B) \Psi_{\mu}(\mathbf{x}, t)+\sum_{\nu \kappa \lambda} \int d \mathbf{y} \Psi_{v}^{*}(\mathbf{y}, t) V_{\{\mu \nu\},\{\kappa \lambda\}}(\mathbf{x}-\mathbf{y})\left[\Phi_{\kappa \lambda}(\mathbf{x}, \mathbf{y}, t)+\Psi_{\kappa}(\mathbf{x}, t) \Psi_{\lambda}(\mathbf{y}, t)\right], \\
i \hbar \frac{\partial}{\partial t} \Phi_{\mu \nu}(\mathbf{x}, \mathbf{y}, t) & =\sum_{\kappa \lambda}\left[H_{\{\mu \nu\},\{\kappa \lambda\}}^{2 \mathrm{~B}}(B) \Phi_{\kappa \lambda}(\mathbf{x}, \mathbf{y}, t)+V_{\{\mu \nu\},\{\kappa \lambda\}}(\mathbf{x}-\mathbf{y}) \Psi_{\kappa}(\mathbf{x}, t) \Psi_{\lambda}(\mathbf{y}, t)\right] .
\end{aligned}
$$

Here $H_{\{\mu \nu\},\{\kappa \lambda\}}^{2 \mathrm{~B}}(B)=\left[H_{\mu}^{1 \mathrm{~B}}(B)+H_{v}^{1 \mathrm{~B}}(B)\right] \delta_{\kappa \mu} \delta_{\lambda v}+V_{\{\mu \nu\},\{\kappa \lambda\}}$ is the Hamiltonian matrix of two atoms. All the other correlation functions can be expressed in terms of the mean field and the pair function [28]. The coupled equations (2) and (3) are the most general form of the first order microscopic quantum dynamics approach of Refs. [28, 32], but they also include the two level mean field approach of Refs. [25, 29, 30] in the Markov approximation [23]. Higher order approximations [40] in the general truncation scheme [32] include few-body inelastic loss phenomena, like three-body recombination [41]. We shall focus in this work on the dominant two-body scattering in the presence of the surrounding Bose gas. 


\section{RESONANCE ENHANCED BINARY COLLISIONS}

\section{A. General properties of Feshbach resonances in ultracold collisions}

We denote the open $s$ wave binary scattering channel of two asymptotically free ${ }^{23} \mathrm{Na}$ condensate atoms in the $(F=$ $1, m_{F}=1$ ) state as the $\{1,1\}$ open channel (or simply as the open channel) with an associated background scattering potential $V_{\{1,1\},\{1,1\}}(\mathbf{r})=V_{\mathrm{bg}}(r)$, where $r$ is the internuclear distance. Analogously, for ${ }^{85} \mathrm{Rb}$ the background scattering potential is denoted by $V_{\{-2,-2\},\{-2,-2\}}(\mathbf{r})=V_{\mathrm{bg}}(r)$. Throughout this paper we shall choose the zero of energy as the dissociation threshold of $V_{\mathrm{bg}}(r)$, i.e. $V_{\mathrm{bg}}(r) \underset{r \rightarrow \infty}{\sim} 0$. Figure 1 shows, for the case of the ${ }^{85} \mathrm{Rb}$ Feshbach resonance, that in the vicinity of the range of magnetic field strengths (from 162.2 to $132 \mathrm{G})$ in Ref. [2] the energy $E_{\mathrm{res}}(B)$ of a closed channel vibrational state (a Feshbach resonance level) $\phi_{\text {res }}(r)$ crosses zero (at $B_{\text {res }}=164.1 \mathrm{G}$ ). The resulting strong coupling between the open and the closed channel is accompanied by the emergence of a highly excited multichannel vibrational molecular bound state $\phi_{\mathrm{b}}$, whose binding energy $E_{\mathrm{b}}(B)$ vanishes at the measurable position of the Feshbach resonance of $B_{0}=154.9 \mathrm{G}$. When the magnetic field strength is swept across the resonance from above, i.e. the energy of the Feshbach resonance level $\phi_{\text {res }}(r)$ is tuned upward, $\phi_{\mathrm{b}}$ thus ceases to exist (see Fig. 11). The $s$ wave scattering length of two asymptotically free condensate atoms, therefore, has a singularity at $B_{0}$. The emergence of a multichannel diatomic bound state, which causes the singularity of the scattering length at $B_{0}$, is the decisive feature of all Feshbach resonances in ultracold collision physics.

\section{B. Two channel approach}

The coupling between the $\{-2,-2\}$ open channel of ${ }^{85} \mathrm{Rb}$ and other open channels, leading, e.g., to inelastic two-body losses, is weak [41]. In the case of ${ }^{23} \mathrm{Na}$ the condensate atoms are in the electronic ground state and inelastic two-body losses are ruled out from the outset. It thus turns out that for the Feshbach resonances of both species the Hamiltonian of the relative motion of two atoms in free space can be reduced to just two channels [22, 43]:

$$
\begin{aligned}
& H_{2 \mathrm{~B}}(B)=-\frac{\hbar^{2}}{m} \nabla^{2}[|\mathrm{bg}\rangle\langle\mathrm{bg}|+| \mathrm{cl}\rangle\langle\mathrm{cl}|]+V_{2 \mathrm{~B}}(B), \\
& V_{2 \mathrm{~B}}(B)=(|\mathrm{bg}\rangle,|\mathrm{cl}\rangle)\left(\begin{array}{cc}
V_{\mathrm{bg}}(r) & W(r) \\
W(r) & V_{\mathrm{cl}}(B, r)
\end{array}\right)\left(\begin{array}{c}
\langle\mathrm{bg}| \\
\langle\mathrm{cl}|
\end{array}\right) .
\end{aligned}
$$

Here $|b g\rangle$ is the product of hyperfine states associated with the open channel, $|\mathrm{cl}\rangle$ is the superposition of products of atomic hyperfine states associated with the strongly coupled closed channel [22], and $W(r)$ couples the two channels. $V_{\mathrm{cl}}(B, r)$ is the closed channel potential that supports the resonance state $\phi_{\text {res }}(r)$, i.e.

$$
\left[-\hbar^{2} \nabla^{2} / m+V_{\mathrm{cl}}(B, r)\right] \phi_{\mathrm{res}}(r)=E_{\mathrm{res}}(B) \phi_{\mathrm{res}}(r) .
$$

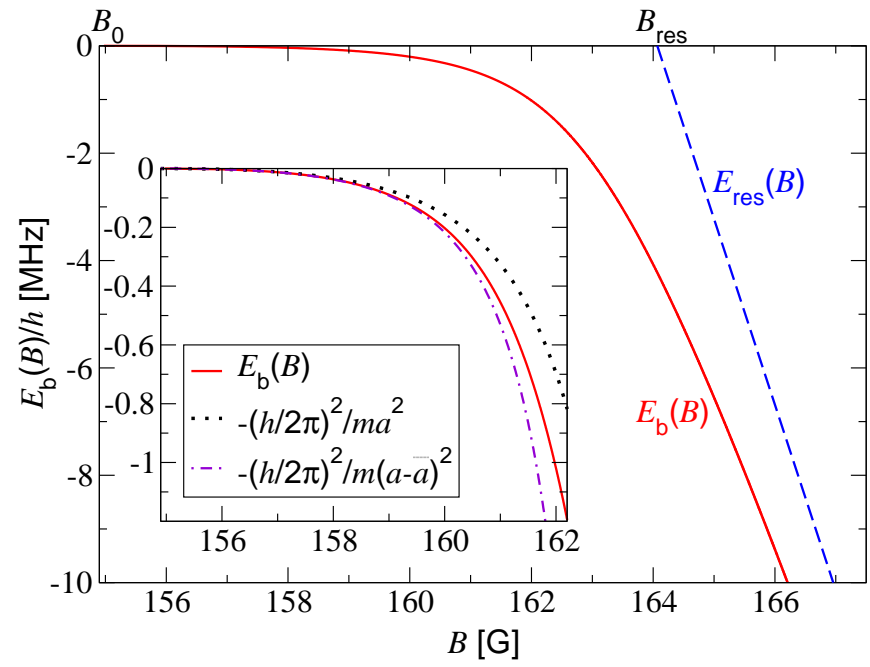

FIG. 1: Magnetic field dependence of the binding energy $E_{\mathrm{b}}(B)$ of the highest excited multichannel vibrational bound state $\phi_{\mathrm{b}}$ of ${ }^{85} \mathrm{Rb}_{2}$ (solid curve) and the energy $E_{\text {res }}(B)$ of the closed channel resonance state $\phi_{\text {res }}(r)$ (dashed curve). $E_{\text {res }}(B)$ depends linearly on $B$ with $h^{-1} d E_{\text {res }} / d B=-3.46 \mathrm{MHz} / \mathrm{G}$. The inset shows $E_{\mathrm{b}}(B)$ (solid curve) as compared to the universal asymptotic formula $-\hbar^{2} / m a^{2}$ for the near resonant binding energy (dotted curve) and its first improvement [42] that accounts for the length scale set by the van der Waals interaction in a single channel picture (dotted dashed curve).

As only the resonance state couples strongly to the open channel, $-\hbar^{2} \nabla^{2} / m+V_{\mathrm{cl}}(B, r)$ can be replaced by the one dimensional Hamiltonian $\left|\phi_{\text {res }}\right\rangle E_{\text {res }}(B)\left\langle\phi_{\text {res }}\right|$ to an excellent approximation [23]. Within the limited range of magnetic field strengths under consideration the energy associated with the resonance state is linear in the magnetic field strength, i.e.

$$
E_{\mathrm{res}}(B)=\left[\frac{d E_{\mathrm{res}}}{d B}\left(B_{\mathrm{res}}\right)\right]\left(B-B_{\mathrm{res}}\right) .
$$

Here $d E_{\text {res }} / d B$ denotes the difference between the magnetic moment of the resonance state and an atom pair in the open channel, and is usually referred to as the slope of the resonance.

\section{Effective low energy potentials}

The typical de Broglie wave lengths of the condensate atoms are much larger than the length scales set by the spatial extent of the binary interactions. The details of the potentials are, therefore, not resolved in the binary collisions. Consequently, we can use the approach of Ref. [23] whose parameters for the ${ }^{23} \mathrm{Na}$ and ${ }^{85} \mathrm{Rb}$ Feshbach resonances we shall briefly summarise. For the potential energy operator $V_{\mathrm{bg}}$ we use the separable form

$$
V_{\mathrm{bg}}=\left|\chi_{\mathrm{bg}}\right\rangle \xi_{\mathrm{bg}}\left\langle\chi_{\mathrm{bg}}\right|
$$


with the convenient Gaussian ansatz

$$
\chi_{\mathrm{bg}}(r)=\frac{e^{-r^{2} / 2 \sigma_{\mathrm{bg}}^{2}}}{\left(\sqrt{2 \pi} \sigma_{\mathrm{bg}}\right)^{3}},
$$

as introduced in Ref. [28] to describe the low energy background scattering of ${ }^{23} \mathrm{Na}$ [22] and ${ }^{85} \mathrm{Rb}$ [3, 4, 36, 37]. The background scattering potential $V_{\mathrm{bg}}$ then recovers the background scattering length of the atoms in the vicinity of the respective resonance, as well as the length scale

$$
l_{\mathrm{vdW}}=\frac{1}{2}\left(m C_{6} / \hbar^{2}\right)^{1 / 4}
$$

set by the van der Waals interaction $-C_{6} / r^{6}$, which is determined by the dispersion coefficient $C_{6}$. Table $\amalg$ provides the relevant physical parameters associated with the Feshbach resonances considered in this paper, and Table III shows the parameters $\xi_{\text {bg }}$ and $\sigma_{\text {bg }}$ characterising the low energy background scattering potential. To recover the widths $\Delta B$ of the resonances and the shifts $B_{0}-B_{\text {res }}$ (see Fig. 1) as given in Table $\llbracket$ it is sufficient to specify $W(r) \phi_{\text {res }}(r)$. We have used the ansatz.

$$
W(r) \phi_{\mathrm{res}}(r)=\zeta \frac{e^{-r^{2} / 2 \sigma^{2}}}{(\sqrt{2 \pi} \sigma)^{3}} .
$$

The parameters $\zeta$ and $\sigma$ can be obtained from Table The scattering length is then exactly given by the formula

$$
a(B)=a_{\mathrm{bg}}\left(1-\frac{\Delta B}{B-B_{0}}\right) \text {. }
$$

The binding energies $E_{\mathrm{b}}(B)$ in Fig. 11 have been obtained with this low energy potential matrix $V_{2 \mathrm{~B}}(B)$ by

$$
H_{2 \mathrm{~B}} \phi_{\mathrm{b}}=E_{\mathrm{b}} \phi_{\mathrm{b}} \text {. }
$$

Figure 2 shows a comparison of $E_{\mathrm{b}}(B)$, for the $907 \mathrm{G}$ Feshbach resonance of ${ }^{23} \mathrm{Na}$, between the low energy potentials in this work and the highly accurate two channel model of Ref. [22]. The associated two-body time evolution operator $U_{2 \mathrm{~B}}(t, \tau)$ can be obtained by relatively simple means [28], even for the time dependent magnetic field strengths in Refs. [2, 21]. $U_{2 \mathrm{~B}}(t, \tau)$ will serve as an input to the solution of the coupled equations (2) and (3).

\section{Differences between the ${ }^{23} \mathrm{Na}$ and ${ }^{85} \mathrm{Rb}$ Feshbach resonances}

Although the ${ }^{23} \mathrm{Na}$ and ${ }^{85} \mathrm{Rb}$ Feshbach resonances considered in this paper can all be described by a low energy two channel Hamiltonian of the form of Eq. (4), with a single resonance state, the multichannel bound states which cause the singularities of the scattering length differ decisively among the resonances within comparable ranges of the magnetic field

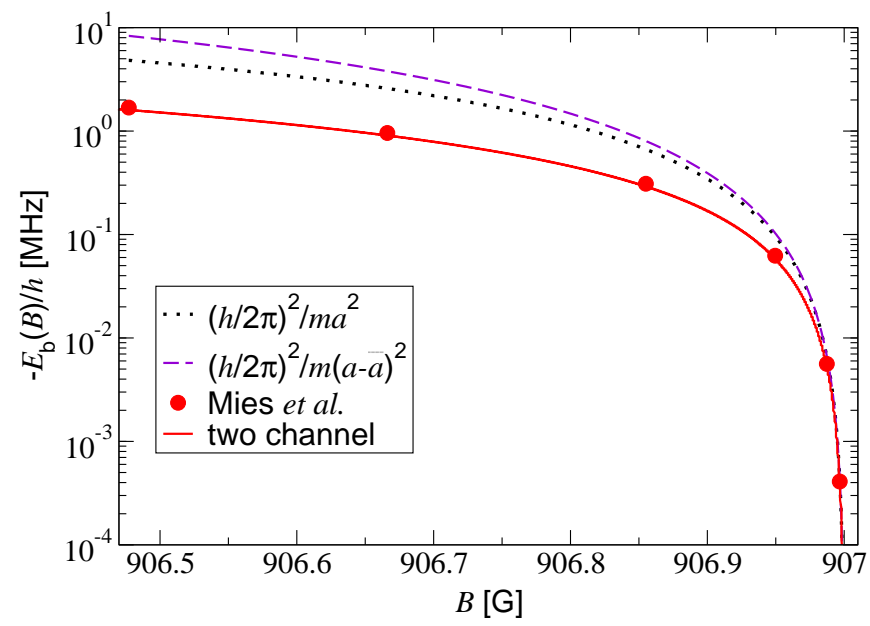

FIG. 2: Magnetic field dependence of the modulus of the binding energy of the highest excited multichannel vibrational bound state of ${ }^{23} \mathrm{Na}_{2}$ in the vicinity of the $907 \mathrm{G}$ Feshbach resonance. The figure compares the binding energies supported by the two channel low energy potential matrix of Ref. [23] and this work (solid curve) with the highly accurate two channel model of Ref. [22] (filled circles). The dotted curve indicates the universal asymptotic formula $E_{\mathrm{b}}=-\hbar^{2} / m a^{2}$ and the dashed curve is the Gribakin and Flambaum [42] estimate that accounts for the length scale set by the van der Waals interaction in a single channel picture. We note that the binding energies are given on a logarithmic scale.

strength. The differences are illustrated in Fig. 3, which shows the admixture $\left|\left\langle\phi_{\text {res }}, \mathrm{cl} \mid \phi_{\mathrm{b}}\right\rangle\right|^{2}$ of the closed channel state to the two channel vibrational molecular bound state $\phi_{\mathrm{b}}$ of the Hamiltonian (4). For ${ }^{85} \mathrm{Rb}$ the admixture stays small $(<30$ $\%)$ over the entire range of experimental [2] magnetic field strengths from 154.9 to $162.2 \mathrm{G}$ on the high field side of the Feshbach resonance (cf., also, Ref. [43]). Even for the broader $(\Delta B=1 \mathrm{G})$ resonance of ${ }^{23} \mathrm{Na}$ at $907 \mathrm{G}$, however, the multichannel bound state $\phi_{\mathrm{b}}$ is transferred immediately into the closed channel resonance state $\phi_{\text {res }}(r)$, with an admixture of more than $80 \%$ within the resonance width. The single channel nature of the ${ }^{85} \mathrm{Rb} 155 \mathrm{G}$ Feshbach resonance is also visible in the binding energies in Fig. 11 because the near resonant single channel Gribakin and Flambaum estimate [28, 42]

$$
E_{\mathrm{b}}=-\hbar^{2} / m(a-\bar{a})^{2}
$$

is applicable, where

$$
\bar{a}=\frac{1}{\sqrt{2}} \frac{\Gamma(3 / 4)}{\Gamma(5 / 4)} l_{\mathrm{vdW}}
$$

is the mean scattering length [42], which accounts for the long range behaviour $-C_{6} / r^{6}$ of the background scattering potential in terms of the van der Waals length in Eq. (10). For the $907 \mathrm{G}$ Feshbach resonance of ${ }^{23} \mathrm{Na}$, however, Fig. 2] shows that Eq. (14) is even less accurate than the universal near resonant estimate [3, 23, 43] $E_{\mathrm{b}}=-\hbar^{2} / m a^{2}$ of the binding energy.

The reason for the single channel nature of the ${ }^{85} \mathrm{Rb} 155 \mathrm{G}$ Feshbach resonance is the large shift of $B_{0}-B_{\text {res }}=-9.2 \mathrm{G}$ 


\begin{tabular}{|c|c|c|c|c|c|c|c|}
\hline Element & $a_{\mathrm{bg}}\left[a_{\mathrm{B}}\right]$ & $C_{6}$ [a.u.] & $E_{-1}[\mathrm{MHz} h]$ & $B_{0}[\mathrm{G}]$ & $\Delta B[\mathrm{G}]$ & $B_{0}-B_{\text {res }}[\mathrm{G}]$ & $\partial E_{\mathrm{res}} / \partial B[\mathrm{MHz} \mathrm{h} / \mathrm{G}]$ \\
\hline${ }^{23} \mathrm{Na}$ & 63.9 [22] & 1561 [44] & 207.8 [45] & 853 [21] & $0.01[22]$ & $-5.85 \times 10^{-3}[46]$ & $5.24[22]$ \\
\hline & $62.8[22]$ & $1561[44]$ & $207.8[45]$ & 907 [21] & $1.0[22]$ & $-0.55[46]$ & $5.24[22]$ \\
\hline${ }^{85} \mathrm{Rb}$ & $-450[3]$ & $4660[36]$ & & $155[36]$ & $11.0[36]$ & $-9.2[46]$ & $-3.46[22]$ \\
\hline
\end{tabular}

TABLE I: Parameters characterising the Feshbach resonances $\left(a_{\text {Bohr }}=0.052918 \mathrm{~nm} ; C_{6}: 1\right.$ a.u. $\left.=0.095734 \times 10^{-24} \mathrm{~J} \mathrm{~nm}^{6}\right)$.

\begin{tabular}{lccccc} 
Element & $B_{0}[\mathrm{G}]$ & $\xi_{\text {bg }}\left[4 \pi \hbar^{2} a_{\text {Bohr }} / m\right]$ & $\sigma_{\text {bg }}\left[a_{\text {Bohr }}\right]$ & $\zeta\left[\mathrm{GHz} h a_{\text {Bohr }}^{3 / 2}\right]$ & $\sigma\left[a_{\text {Bohr }}\right]$ \\
\hline${ }^{23} \mathrm{Na}$ & 853 & -197 & 27.2 & -3.96 & 14.5 \\
& 907 & -186 & 26.5 & -44.0 & 17.4 \\
${ }^{85} \mathrm{Rb}$ & 155 & -99.3 & 71.9 & 19.0 & 52.4
\end{tabular}

TABLE II: Parameters determining the low energy two channel potential matrix.

(see Fig.11, which leads to a large detuning of $E_{\text {res }}\left(B_{0}\right) / h=32$ $\mathrm{MHz}$ at the resonance position. The naive physical two level picture, in which the resonance level $\phi_{\text {res }}(r)$ is swept across the zero energy level of the background scattering potential at the measurable position $B_{0}$ of the Feshbach resonance, and is then transferred to a bound molecular state $\phi_{\mathrm{b}}$ at negative energies $E_{\text {res }}<0$ (see, e.g., Ref. [46]), is therefore inapplicable. In fact, the resonance state does not even cross the threshold within the entire range of experimental magnetic field strengths (from 162.2 to $132 \mathrm{G}$ ) in Ref. [2].

\section{MANY-BODY APPROACHES TO FESHBACH RESONANCE CROSSING EXPERIMENTS}

\section{A. Non-Markovian nonlinear Schrödinger equation}

Inserting the two-channel Hamiltonian (4) into Eq. (3) implies that the pair function has a closed channel component $\Phi_{\mathrm{cl}}(\mathbf{x}, \mathbf{y}, t)$, proportional to $\phi_{\text {res }}(r)$, and a long range component $\Phi_{\mathrm{bg}}(\mathbf{x}, \mathbf{y}, t)$ in the open channel. Only the $\left(F=2, m_{F}=\right.$ -2) hyperfine state of ${ }^{85} \mathrm{Rb}$ and the $\left(F=1, m_{F}=1\right)$ hyperfine state of ${ }^{23} \mathrm{Na}$ have a mean field that we will denote simply by $\Psi(\mathbf{x}, t)$. The dynamic equation (3) can be solved formally in terms of the mean field and inserted into Eq. (2) [28, 32]. In the first order microscopic quantum dynamics approach the entire dynamics of the gas is then described by a single nonlinear Schrödinger equation [23, 28, 32, 47]

$$
\begin{aligned}
i \hbar \frac{\partial}{\partial t} \Psi(\mathbf{x}, t)= & {\left[-\frac{\hbar^{2}}{2 m} \nabla^{2}+V_{\text {trap }}(\mathbf{x})\right] \Psi(\mathbf{x}, t) } \\
& -\Psi^{*}(\mathbf{x}, t) \int_{t_{0}}^{\infty} d \tau \Psi^{2}(\mathbf{x}, \tau) \frac{\partial}{\partial \tau} h(t, \tau),
\end{aligned}
$$

with

$$
h(t, \tau)=(2 \pi \hbar)^{3}\left\langle 0, \mathrm{bg}\left|V_{2 \mathrm{~B}}(B(t)) U_{2 \mathrm{~B}}(t, \tau)\right| 0, \mathrm{bg}\right\rangle \theta(t-\tau) .
$$

Here $U_{2 \mathrm{~B}}(t, \tau)$ is the time evolution operator associated with the magnetic field dependent Hamiltonian (4), $\theta(t-\tau)$ is the step function, which yields unity for $t>\tau$ and vanishes elsewhere, and $|0, \mathrm{bg}\rangle$ is the zero momentum plane wave of the relative motion of two atoms in the open channel. We note that Eq. 16 accounts for the entire two-body collision dynamics including the continuum of modes above the dissociation threshold of the background scattering potential in a non-perturbative manner.

\section{B. Contributions beyond mean field approaches}

The solution of Eq. (16) determines the pair function, via Eq. (3), through its components in the open and closed channels:

$$
|\Phi(t)\rangle=|\mathrm{bg}\rangle \Phi_{\mathrm{bg}}(t)+|\mathrm{cl}\rangle \Phi_{\mathrm{cl}}(t) .
$$

A derivation [23, 28] beyond the scope of this paper shows that the pair function determines the components of the density matrix of the non-condensed fraction in the open and closed channel to be

$$
\Gamma_{\mathrm{bg}}\left(\mathbf{x}, \mathbf{x}^{\prime}, t\right)=\int d \mathbf{y} \Phi_{\mathrm{bg}}(\mathbf{x}, \mathbf{y}, t) \Phi_{\mathrm{bg}}^{*}\left(\mathbf{x}^{\prime}, \mathbf{y}, t\right)
$$

and

$$
\Gamma_{\mathrm{cl}}\left(\mathbf{x}, \mathbf{x}^{\prime}, t\right)=\int d \mathbf{y} \Phi_{\mathrm{cl}}(\mathbf{x}, \mathbf{y}, t) \Phi_{\mathrm{cl}}^{*}\left(\mathbf{x}^{\prime}, \mathbf{y}, t\right),
$$

respectively. The density of the gas is thus given by

$$
n(\mathbf{x}, t)=\Gamma_{\mathrm{bg}}(\mathbf{x}, \mathbf{x}, t)+\Gamma_{\mathrm{cl}}(\mathbf{x}, \mathbf{x}, t)+|\Psi(\mathbf{x}, t)|^{2},
$$

and the total number of atoms $N=\int d \mathbf{x} n(\mathbf{x}, t)$ is a constant of motion [23, 28].

In general, the solutions of the coupled equations (2) and (3) depend not only on the initial condensate wave function $\Psi\left(\mathbf{x}, t_{0}\right)$ and but also on the initial pair function $\Phi\left(t_{0}\right)$, which has so far been neglected in Eq. 16. These initial conditions are determined by the experimental situation. For Feshbach resonance crossing experiments with ${ }^{23} \mathrm{Na}$ it appears reasonable to require the condensate density to be stationary in the absence of any variation of the magnetic field strength. To find the stationary solution of the coupled equations (2) and (3), we assume that the magnetic field strength at time $t_{0}$ is sufficiently far away from the Feshbach resonance that the binary dynamics is determined entirely by the background scattering. The 

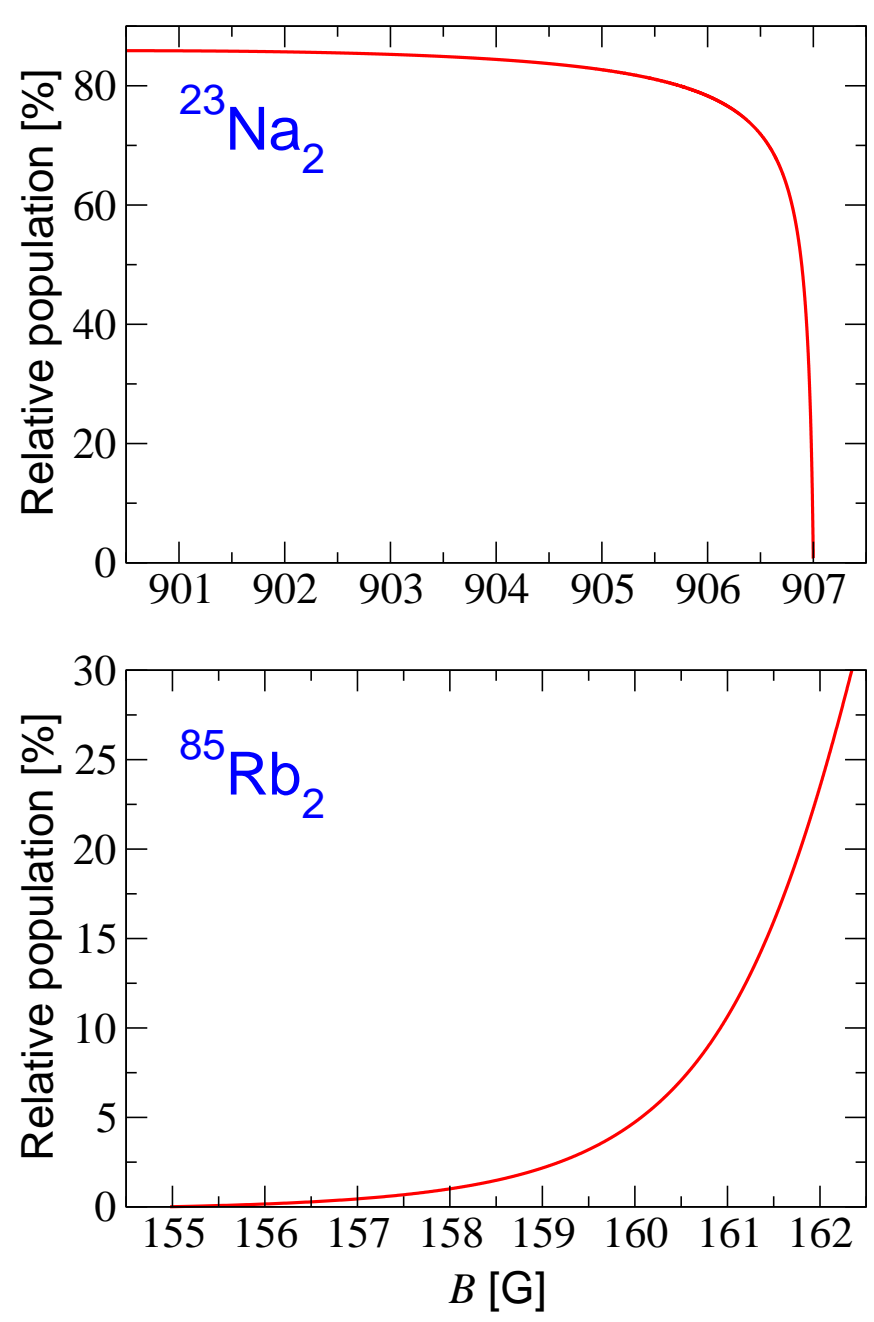

FIG. 3: Magnetic field dependence of the population of the closed channel component of the highest excited vibrational bound state $\phi_{\mathrm{b}}$ associated with the ${ }^{23} \mathrm{Na} 907 \mathrm{G}$ and the ${ }^{85} \mathrm{Rb} 155 \mathrm{G}$ Feshbach resonance. The populations were determined from the two channel Hamiltonian (4).

closed channel component of the initial pair function can then be neglected, and for its open channel component $\Phi_{\mathrm{bg}}$ as well as for the mean field $\Psi$ we choose the ansatz:

$$
\begin{aligned}
\Psi(\mathbf{R}, t) & =\sqrt{n_{0}(\mathbf{R})} e^{-i \mu_{0}\left(t-t_{0}\right) / \hbar}, \\
\Phi_{\mathrm{bg}}(\mathbf{R}, \mathbf{r}, t) & =\Phi_{\mathrm{bg}}\left(\mathbf{R}, \mathbf{r}, t_{0}\right) e^{-i 2 \mu_{0}\left(t-t_{0}\right) / \hbar} .
\end{aligned}
$$

Here $\mathbf{R}=(\mathbf{x}+\mathbf{y}) / 2$ and $\mathbf{r}=\mathbf{y}-\mathbf{x}$ are centre of mass and relative coordinates, respectively, $n_{0}$ is the equilibrium condensate density, and $\mu_{0}$ can be interpreted as the chemical potential. The formal solution of Eq. (3) then provides the general form of the stationary pair function

$$
\Phi_{\mathrm{bg}}\left(\mathbf{R}, \mathbf{r}, t_{0}\right)=n_{0}(\mathbf{R})(2 \pi \hbar)^{3 / 2} \operatorname{Re}\left[\left\langle\mathbf{r}\left|G_{\mathrm{bg}}\left(2 \mu_{0}+i 0\right) V_{\mathrm{bg}}\right| 0\right\rangle\right],
$$

where

$$
G_{\mathrm{bg}}\left(2 \mu_{0}+i 0\right)=\left[2 \mu_{0}+i 0+\hbar^{2} \nabla^{2} / m-V_{\mathrm{bg}}\right]^{-1}
$$

denotes the energy dependent Green's function associated with the background scattering potential $V_{\mathrm{bg}}$. Inserting the stationary ansatz of Eq. (22) for the mean field into Eq. (2) then yields the stationary nonlinear Schrödinger equation:

$$
\begin{aligned}
\mu_{0} \sqrt{n_{0}(\mathbf{x})}= & {\left[-\frac{\hbar^{2}}{2 m} \nabla^{2}+V_{\text {trap }}(\mathbf{x})\right] \sqrt{n_{0}(\mathbf{x})} } \\
& +\left[n_{0}(\mathbf{x})\right]^{3 / 2}(2 \pi \hbar)^{3} \operatorname{Re}\left[\left\langle 0\left|T_{\text {bg }}\left(2 \mu_{0}+i 0\right)\right| 0\right\rangle\right] .
\end{aligned}
$$

Here $T_{\mathrm{bg}}\left(2 \mu_{0}+i 0\right)$ is the energy dependent $T$ matrix associated with the background scattering potential $V_{\mathrm{bg}}$ (see, e.g., Ref. [32]). Evaluating the $T$ matrix at the energy $2 \mu_{0} \rightarrow 0$, Eq. (26) recovers the usual Gross-Pitaevskii equation in the dilute gas limit ( $n_{\mathrm{pk}} a_{\mathrm{bg}}^{3} \ll 1$, where $n_{\mathrm{pk}}$ is the peak density), which then determines the chemical potential $\mu_{0}$ as well as the stationary condensate density $n_{0}(\mathbf{x})$. In the experiments with ${ }^{23} \mathrm{Na}$ [21] the peak condensate density is on the order of $n_{\mathrm{pk}} \approx 1.5 \times 10^{15} \mathrm{~cm}^{-3}$ and including the initial pair function influences the dynamics on the time scales of the passage across the Feshbach resonances. We have thus included the initial pair function in Eq. (16) as explained in Ref. [32]. In the experiments with ${ }^{85} \mathrm{Rb}$ the peak density $n_{\mathrm{pk}} \approx 2.2 \times 10^{12} \mathrm{~cm}^{-3}$ turns out to be sufficiently low for initial pair correlations to be negligible.

\section{Two level mean field approach}

The two level mean field approach of Refs. [25, 29, 30] can be derived from the first order microscopic quantum dynamics approach in Eqs. (2) and (3) with the ansatz

$$
\Phi_{\mathrm{cl}}(\mathbf{R}, \mathbf{r}, t)=\sqrt{2} \phi_{\mathrm{res}}(r) \Psi_{\mathrm{res}}(\mathbf{R}, t)
$$

for the closed channel component of the pair function in centre of mass and relative coordinates. Here $\phi_{\text {res }}(r)$ is the closed channel resonance level. A derivation [23] beyond the scope of this paper then shows that Eqs. (2) and (3) yield the coupled equations

$$
\begin{aligned}
i \hbar \frac{\partial}{\partial t} \Psi(\mathbf{x}, t)= & {\left[-\frac{\hbar^{2}}{2 m} \nabla^{2}+\frac{m}{2} \omega_{\text {ho }}^{2}|\mathbf{x}|^{2}\right] \Psi(\mathbf{x}, t) } \\
& +g_{\text {bg }}|\Psi(\mathbf{x}, t)|^{2} \Psi(\mathbf{x}, t) \\
& +g_{\text {res }} \Psi^{*}(\mathbf{x}, t) \sqrt{2} \Psi_{\text {res }}(\mathbf{x}, t)
\end{aligned}
$$

for the condensate mean field, and

$$
\begin{aligned}
i \hbar \frac{\partial}{\partial t} \Psi_{\text {res }}(\mathbf{R}, t)= & {\left[-\frac{\hbar^{2}}{4 m} \nabla^{2}+\frac{2 m}{2} \omega_{\text {ho }}^{2}|\mathbf{R}|^{2}\right] \Psi_{\text {res }}(\mathbf{R}, t) } \\
& +\left[\frac{d E_{\text {res }}}{d B}\left(B_{\text {res }}\right)\right]\left[B(t)-B_{0}\right] \Psi_{\text {res }}(\mathbf{R}, t) \\
& +\frac{1}{\sqrt{2}} g_{\text {res }} \Psi^{2}(\mathbf{R}, t)
\end{aligned}
$$

for the mean field associated with the resonance level in the Markov approximation. Here we have assumed that the atom 
trap is spherical and that the closed channel resonance state experiences twice the trap potential of the atoms. The coupling constants $g_{\text {bg }}$ and $g_{\text {res }}$ are given by $g_{\text {bg }}=4 \pi \hbar^{2} a_{\text {bg }} / m$ and $g_{\text {res }}=(2 \pi \hbar)^{3 / 2}\left\langle\phi_{\text {res }}|W| \phi_{0}^{(+)}\right\rangle$, where $a_{\text {bg }}$ is the background scattering length, and $\phi_{0}^{(+)}(\mathbf{r})$ is the zero energy wave function associated with the background scattering [23]. The physical picture underlying the two level mean field approach (see, e.g., Ref. [46]) can be described in terms of a linear curve crossing between the energy $E_{\mathrm{res}}(B)$ associated with the closed channel resonance level $\phi_{\text {res }}(r)$ and the zero energy level of two condensate atoms (see Fig. 11. This picture, however, neglects all the other modes of the two-body background scattering continuum and, consequently, does not recover the nature of the highest excited vibrational diatomic bound state [43]. Even more importantly, in the context of this paper, the absence of continuum modes also implies that the two level mean field approach is unsuited to describe the energy distribution of the atoms lost from the condensate in an upward ramp of the Feshbach resonance level.

\section{COMPARISON BETWEEN DIFFERENT MANY-BODY APPROACHES}

\section{A. Loss of condensate atoms in upward ramps of the resonance level}

Since, in accordance with Eqs. (19) and (20), the noncondensate density stems directly from the pair function, the build-up of pair correlations is the dominant source of atom loss from the condensate. In general, a time dependent magnetic field can transfer pairs of trapped condensate atoms either into highly excited diatomic molecules or into the complementary part of the two-body energy spectrum that contains a quasi continuum of excited levels [28]. In the experiments in Refs. [2, 21] molecular formation can be ruled out because the linear ramps start on the side of the Feshbach resonance where the scattering length is positive and cross the resonance in such a way that the highest excited multichannel vibrational bound state $\phi_{\mathrm{b}}$ ceases to exist during the passage (see Fig. 11 [48]. The remaining source of atom loss from the condensate is the formation of correlated pairs of atoms in initially unoccupied excited energy levels. As the time dependent homogeneous magnetic field provides only energy but no momentum the centre of mass motion of these pairs is limited by the initial momentum spread of the Bose-Einstein condensed gas. Their comparatively fast relative motion, however, has been observed as a very dilute burst of atoms, e.g., in Ref. [3], and the typical scale of experimental single particle kinetic energies of $E_{\mathrm{kin}} / k_{\mathrm{B}} \sim 150 \mathrm{nK}$ has been confirmed with the first order microscopic quantum dynamics approach of this work [28]. We note that the two level mean field approach can only describe the transfer of condensate atoms into the resonance level $\phi_{\text {res }}(r)$. The subsequent decay of this metastable state has been modelled in Ref. [25] in terms of a constant decay width. Corrections to this approach, which account for the energy dependence of this decay width have been provided in Refs. [15, 23].

\section{B. Experimental conditions}

We have solved Eq. (16) and Eqs. (28) and 29) using a cylindrical trap potential with the radial (axial) trap frequencies of $v_{r}=150 \mathrm{~Hz}\left(v_{z}=1500 \mathrm{~Hz}\right)$ for the sodium experiments [21], and of $v_{r}=17.5 \mathrm{~Hz}\left(v_{z}=6.8 \mathrm{~Hz}\right)$ in the case of ${ }^{85} \mathrm{Rb}$ [2]. As the initial condensate mean field we have used the ground state of the Gross-Pitaevskii equation with $N=900000{ }^{23} \mathrm{Na}$ atoms [21] and $N=1594{ }^{85} \mathrm{Rb}$ atoms [49], respectively. The initial scattering length was chosen to be the background scattering length $a_{\mathrm{bg}}$ for the ${ }^{23} \mathrm{Na}$ atoms (cf. Table I), and $a=228 a_{\text {Bohr }}$ in the case of ${ }^{85} \mathrm{Rb}$, corresponding to the experimental evaporation field strength of about 162.2 $\mathrm{G}$. Under these initial conditions the condensate is weakly interacting $\left({ }^{23} \mathrm{Na}: n_{\mathrm{pk}} a^{3} \approx 6 \times 10^{-5}\right.$ with a peak density of $n_{\mathrm{pk}} \approx 1.5 \times 10^{15} \mathrm{~cm}^{-3} ;{ }^{85} \mathrm{Rb}: n_{\mathrm{pk}} a^{3} \approx 10^{-6}$ with a peak density of $n_{\mathrm{pk}} \approx 2.2 \times 10^{12} \mathrm{~cm}^{-3}$ ).

Starting from $162.2 \mathrm{G}$, in the case of ${ }^{85} \mathrm{Rb}$, we have studied linear ramps of the magnetic field strength across the $155 \mathrm{G}$ Feshbach resonance down to $132 \mathrm{G}$ [2]. In the course of these studies we have varied the inverse ramp speeds within the experimental range [2] between $1.2 \mu \mathrm{s} / \mathrm{G}$ and $200 \mu \mathrm{s} / \mathrm{G}$. The sodium ramps where extended over much larger timescales in the experiments [21, 50]. We have therefore chosen the ramp intervals in such a way that for each ramp speed the detuning $\left|E_{\text {res }}(B)\right|$ of the closed channel resonance state was sufficiently large at the initial and final times that increasing the ramp intervals did not significantly change the total loss of atoms from the condensate.

\section{Predictions of the different many-body approaches}

Figure 4 shows the theoretical condensate loss curves $(N-$ $\left.\int d \mathbf{x}|\Psi(\mathbf{x}, t)|^{2}\right) / N$ in dependence on the inverse ramp speed for the ${ }^{23} \mathrm{Na}$ Feshbach resonances [51], together with the experimental data from Ref. [21]. In the course of these studies, we have solved Eq. 16 with a variety of different methods. These methods involve complete solutions of Eq. 16 including the potential of a spherical trap with the mean frequency $v_{\text {ho }}=\sqrt[3]{v_{r}^{2} v_{z}}=697 \mathrm{~Hz}$, as well as the local density approximation, which consists in solving Eq. (16) in the homogeneous gas limit with densities weighted according to the initial density distribution associated with the realistic cylindrical atom trap. We have performed these calculations including the two-body time evolution associated with the full two channel Hamiltonian (4) and with the single channel approach described in Refs. [23, 28]. The single channel approach includes the time evolution of two atoms in the open channel with the background scattering potential adjusted in such a way that it recovers, at each magnetic field strength, the scattering length in Eq. (12) as well as the Gribakin and Flambaum estimate of the binding energy $E_{b}$ in Eq. (14). We note that the single channel approach does not explicitly include the closed channel resonance state. For comparison, we have also solved Eqs. (28) and (29) of the two level mean field approach using a spherical trap potential with the mean 

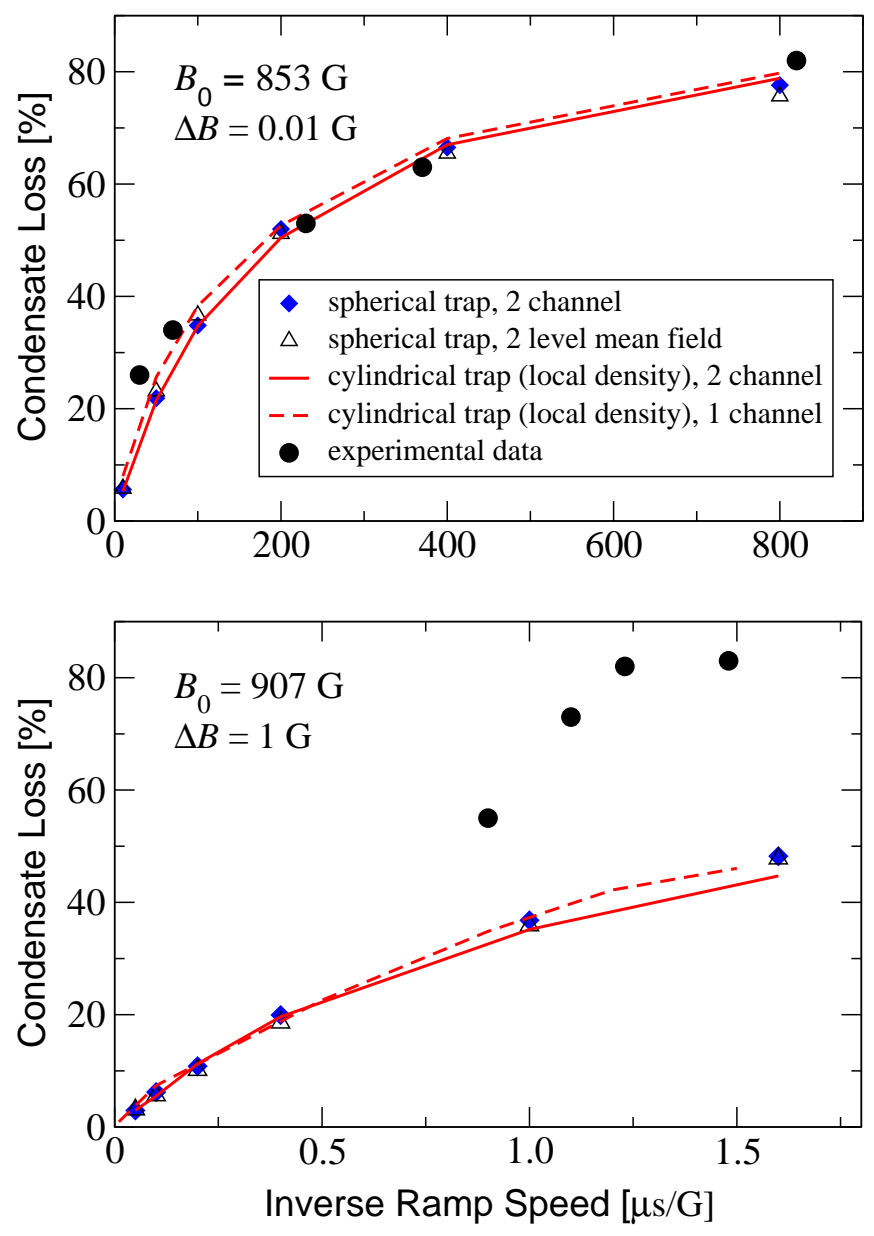

FIG. 4: Loss of atoms from a ${ }^{23} \mathrm{Na}$ condensate at the end of a linear magnetic field ramp across the $853 \mathrm{G}$ and $907 \mathrm{G}$ Feshbach resonances, respectively, as a function of the inverse ramp speed. The filled circles are the experimental data from Ref. [21]. The diamonds are determined from Eq. 16 using a spherical trap potential with the mean experimental trap frequency. The solid curve represents the calculation in the local density approximation, solving Eq. 16) for uniform gases, with densities weighted according to the initial distribution in a cylindrical trap. The dashed curve shows the corresponding result from a single channel calculation as described in Refs. [23, 28]. The triangles correspond to the predictions of the two level mean field approach of Eqs. 28) and 29] with a spherical trap potential.

frequency of the cylindrical trap in the experiment [21].

The comparison between the theoretical predictions shows that although the description of the underlying microscopic collision physics differs decisively among the approaches, the predicted total loss of condensate atoms is always virtually the same. The remnant condensate fractions are therefore largely insensitive not only to phenomena related to the inhomogeneity of realistic Bose-Einstein condensates, like collective excitations, but also to the two channel nature of the two-body energy states at magnetic field strengths in the vicinity of the Feshbach resonances. In particular, the applicability of the single channel approach of Refs. [23, 28] appears surprising

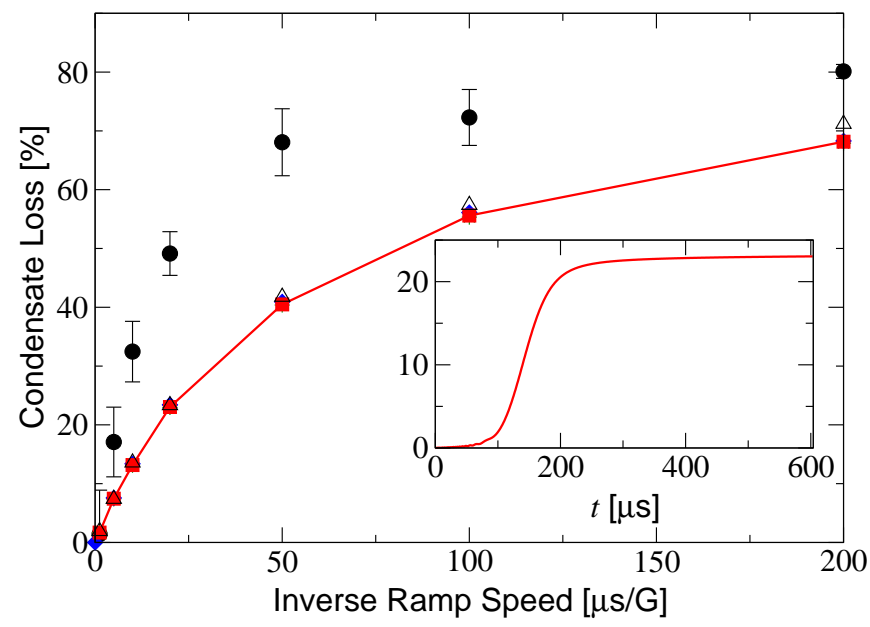

FIG. 5: Loss of atoms from a ${ }^{85} \mathrm{Rb}$ condensate at the end of a linear ramp of the magnetic field strength across the $155 \mathrm{G}$ Feshbach resonance as a function of the inverse ramp speed. The circles with error bars are the experimental data from Ref. [2]. The squares are determined from Eq. 16 using a cylindrical trap potential with the experimental trap frequencies. The diamonds are obtained with a spherical trap potential that accounts only for the mean trap frequency and the stars represent a local density approximation for the cylindrical trap. The triangles correspond to the two level mean field approach of Eqs. (28) and (29) in a spherical trap. The inset shows the dynamics of the loss of condensate atoms in the cylindrical trap for the $20 \mu \mathrm{s} / \mathrm{G}$ ramp.

because the underlying single channel Gribakin and Flambaum estimate [42] of the near resonant binding energy $E_{\mathrm{b}}$ in Eq. (14) is rather poor in the case of the ${ }^{23} \mathrm{Na}$ Feshbach resonances (cf. Fig. 2). The comparison between theory and experiment suggests good agreement for the $853 \mathrm{G}$ Feshbach resonance of ${ }^{23} \mathrm{Na}$ but there are significant deviations for the broader $907 \mathrm{G}$ resonance. Similar observations were reported also in Ref. [25].

Figure 5 shows the analogous theoretical predictions of the different approaches with respect to the loss of condensate atoms $\left(N-\int d \mathbf{x}|\Psi(\mathbf{x}, t)|^{2}\right) / N$ in a gas of ${ }^{85} \mathrm{Rb}$, together with the associated experimental data from Ref. [2], in dependence on the inverse ramp speed. Also in this case, all many-body approaches, including the two level mean field model, yield virtually the same results. This is particularly remarkable because the physical picture of a single linear curve crossing [46], underlying the two level mean field approach, is not applicable to the experiments [2] (cf. Subsection IIID). The predicted monotonic curves in Fig. 5 follow the experimental trend but they consistently underestimate the observed losses in Ref. [2].

\section{UNIVERSALITY OF MOLECULAR DISSOCIATION SPECTRA}

Upward ramps of Feshbach resonance levels also play an important role in the detection of ultracold molecules pro- 


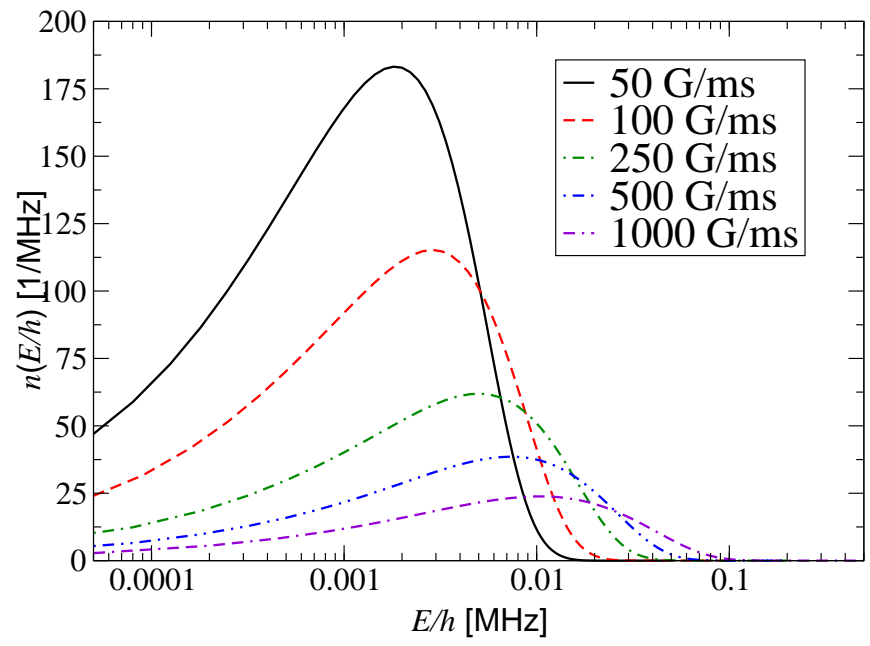

FIG. 6: Dissociation spectra of the highest excited vibrational bound state of ${ }^{85} \mathrm{Rb}_{2}$ as a function of the relative energy of the fragments. The speeds of the linear ramps across the $155 \mathrm{G}$ Feshbach resonance were varied between $50 \mathrm{G} / \mathrm{ms}$ (solid curve) and $1000 \mathrm{G} / \mathrm{ms}$ (double dashed dotted curve). The ramps started from the experimental evaporation magnetic field strength of $B_{i}=162.2 \mathrm{G}$ [2] down to a final magnetic field strength $B_{f}<B_{0}=154.9 \mathrm{G}$, which was chosen sufficiently small that at each ramp speed the spectrum was insensitive to variations of $B_{f}$. The exact calculations based on Eq. 30 were performed with the two channel Hamiltonian (4). We note that the relative kinetic energies of the molecular fragments are given on a logarithmic scale.

duced in atomic Bose-Einstein condensates, as they are frequently used for the purpose of molecular dissociation upon passage across the resonance [6, 7, 15, 38]. The dissociation mechanism can be readily understood from the binding energies in Fig. 1 for the example of ${ }^{85} \mathrm{Rb}$; tuning the magnetic field strength across the resonance position from the high field side adiabatically transfers the bound state $\phi_{\mathrm{b}}$ into the zero energy state of two asymptotically free atoms in the open channel. Starting from the molecular bound state $\phi_{\mathrm{b}}\left(B_{i}\right)$ at the magnetic field strength $B_{i}$, the probability of detecting a free pair of atoms with a relative energy between $E$ and $E+d E$ is given by [23]:

$$
n(E) d E=p^{2} d p \int d \Omega_{\mathbf{p}}\left|\left\langle\phi_{\mathbf{p}}\left(B_{f}\right)\left|U_{2 \mathrm{~B}}\left(t_{f}, t_{i}\right)\right| \phi_{\mathrm{b}}\left(B_{i}\right)\right\rangle\right|^{2} .
$$

Here $\phi_{\mathbf{p}}\left(B_{f}\right)$ is the continuum energy state of the two-body two channel Hamiltonian (4) [23], at the final magnetic field strength $B_{f}$ of the ramp, which is associated with the final relative momentum $\mathbf{p}$ of the atom pair, and $d \Omega_{\mathbf{p}}$ denotes the angular component of $d \mathbf{p}$. We note that the relative kinetic energy of the atoms is related to their relative momentum by $E=p^{2} / m$.

Figure 6 shows the dissociation spectra $n(E)$ as obtained from Eq. (30) for the dissociation of ${ }^{85} \mathrm{Rb}_{2}$ Feshbach molecules in the highest excited multichannel vibrational state $\phi_{\mathrm{b}}$ upon passage across the $155 \mathrm{G}$ Feshbach resonance. In these calculations the initial magnetic field strength was chosen as the experimental evaporation magnetic field strength of

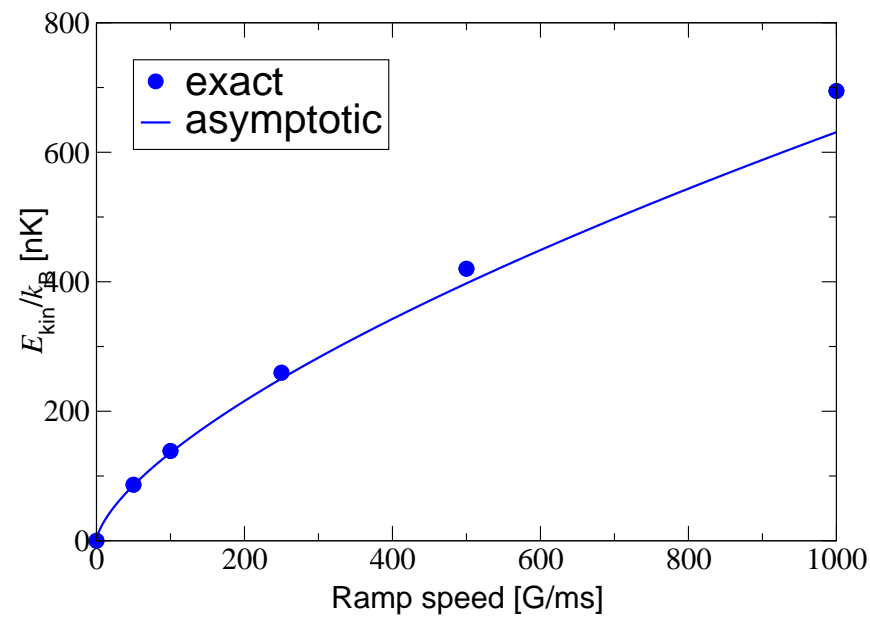

FIG. 7: Mean single particle kinetic energies of the molecular fragments associated with the exact dissociation spectra obtained from Eq. 30] in Fig. 6 (circles) in dependence on the ramp speed. The solid curve has been obtained from the asymptotic prediction in Eq. 32 for comparison. The single particle kinetic energy is half the energy of the relative motion of the fragments in Fig. 6

$B_{i}=162.2 \mathrm{G}$ reported in Ref. [2]. We have varied the speeds of the linear ramps from $50 \mathrm{G} / \mathrm{ms}$ to $1000 \mathrm{G} / \mathrm{ms}$, which illustrates that the dissociation spectra become broader in the energies with increasing ramp speeds.

The mean kinetic energies

$$
\left\langle E_{\mathrm{kin}}\right\rangle=\frac{1}{2} \int_{0}^{\infty} d E \operatorname{En}(E)
$$

of the molecular fragments after the dissociation characterise the speed of expansion of the gas of molecular fragments before the detection in related experiments [6, 7, 15, 38]. We note that the kinetic energy of a single atom is $E_{\text {kin }}=p^{2} / 2 m$, which is half the energy of the relative motion of a pair. A Fermi Golden Rule argument [15] shows that $\left\langle E_{\text {kin }}\right\rangle$ can be expressed in terms of physical parameters of a Feshbach resonance, of the ramp speed $|d B / d t|$ and of Euler's $\Gamma$ function [15, 23]:

$$
\left\langle E_{\mathrm{kin}}\right\rangle=\frac{1}{3}\left[\frac{3}{4} \sqrt{\frac{\hbar^{2}}{m}} \frac{\hbar|d B / d t|}{\left|a_{\mathrm{bg}}\right||\Delta B|}\right]^{2 / 3} \Gamma(2 / 3) .
$$

The derivation of Eq. 32 given in Ref. [15] relies upon the linear curve crossing picture described in Refs. [22, 46], which identifies the stable multichannel molecular bound state $\phi_{\mathrm{b}}$ with the closed channel resonance state $\phi_{\mathrm{res}}(r)$ below the dissociation threshold of the background scattering potential. Above the threshold $\phi_{\text {res }}(r)$ acquires a decay width dependent on the energy $E_{\text {res }}(B)$, which is associated with $\phi_{\text {res }}(r)$. This Fermi Golden Rule decay width leads to the dissociation of the molecules.

We expect Eq. 32 to be accurate provided that the initial and final magnetic field strengths of the linear ramp are sufficiently far from the resonance position. This has 
been demonstrated in the experiments of Ref. [15], in which ${ }^{23} \mathrm{Na}_{2}$ molecules in the highly excited multichannel vibrational bound state $\phi_{\mathrm{b}}$ were dissociated upon passage of the magnetic field strength across the $907 \mathrm{G}$ Feshbach resonance. The derivation in Ref. [15] is obviously inapplicable to ${ }^{85} \mathrm{Rb}$ ramps in the experimental range of magnetic field strengths from 162.2 down to $132 \mathrm{G}$ of Ref. [2] because the resonance level stays always above the threshold and the molecular bound state $\phi_{\mathrm{b}}$ has only a small admixture of $\phi_{\text {res }}(r)(<30$ $\%$ in Fig 3]. Figure 7 shows, however, that the analytic formula (32) is remarkably accurate. Small deviations between Eq. (32) and the exact mean energies obtained from Eqs. (30) and (31) only occur with increasing ramp speeds. The molecular dissociation spectra thus exhibit the same insensitivity to the exact microscopic binary collision physics as the remnant condensate fractions in Section $\mathrm{V}$

\section{CONCLUSIONS}

We have shown in Sections V] and VI that remnant condensate fractions or molecular dissociation spectra associated with linear upward ramps of a Feshbach resonance level do not reveal the details of the underlying microscopic binary collision physics. The formula (32) for the mean single particle kinetic energy of molecular fragments suggests that the Landau Zener parameter [22, 23]

$$
\delta_{\mathrm{LZ}}=4 \pi \frac{N-1}{\mathcal{V}} \frac{\hbar}{m} \frac{\left|a_{\mathrm{bg}}\right||\Delta B|}{|d B / d t|}
$$

determines the universal observables in Sections $[\mathrm{V}$ and $\mathrm{VI}$ when $\left|a_{\mathrm{bg}}\right|,|\Delta B|$ and $|d B / d t|$ are the parameters that can be varied independently. Here $N$ is the number of atoms and $V$ is the volume of the gas. For $N=2$ atoms the Landau Zener coefficient exactly determines the final population in the zero energy level by

$$
p_{0}=\exp \left(-2 \pi \delta_{\mathrm{LZ}}\right)
$$

in a two level linear curve crossing model of a Feshbach resonance [23, 46], provided that the linear ramp starts and ends asymptotically far from the crossing point $B_{\text {res }}$ (see Fig. 1). The analysis of the two level mean field equations 28 and (29) of Ref. [23] reveals that the final populations of the remnant condensate and of the closed channel resonance state are indeed determined by $\delta_{\mathrm{LZ}}$, i.e. the final populations are constant when the parameters $\left|a_{\mathrm{bg}}\right|,|\Delta B|$ and $|d B / d t|$ are varied in such a way that $\delta_{\mathrm{LZ}}$ is kept constant. In particular, the dependence of Eq. 33 on the modulus of the ramp speed shows that the final populations are insensitive with respect to the ramp direction.

The analysis in Ref. [52] shows that Eq. (34) determines the exact asymptotic population $p_{0}$ in the open channel ground state even when the complete quasi continuum of modes in the open channel is taken into account in a linear curve crossing model of a Feshbach resonance. This explains why including the full two channel time evolution operator in Eq. (16) leads to the same results as the two level mean field approach for the ${ }^{23} \mathrm{Na}$ Feshbach resonances in Section $\mathrm{V}$ given that phenomena related to the non Markovian nature of Eq. (16) are negligible. Our studies of the $155 \mathrm{G}$ Feshbach resonance of ${ }^{85} \mathrm{Rb}$ in Figs. 5] and 7 suggest that also the asymptotic criterion of applicability of the two-body Landau Zener approach can be violated to a large extent without any significant changes in the results. We note, however, that the two level picture is not suited to describe the interferometric experiments of Refs. [3, 4], which employ a sequence of magnetic field pulses. In fact, the occupation of binary continuum modes, observed in these experiments, is ruled out in any two level approach from the outset and, in addition, the nature of the highest excited vibrational diatomic bound state is not recovered. This explains why the two level mean field approach fails to predict the experimentally observed atom-molecule Ramsey fringes [39].

Although the Landau Zener coefficient in Eq. (33) can be derived from a linear curve crossing model of a Feshbach resonance, it is independent of the slope $d E_{\text {res }} / d B$ of the resonance and contains just the parameters $a_{\mathrm{bg}}$ and $\Delta B$ of the Feshbach resonance, which determine the scattering length in Eq. (12). In fact, the inset of Fig. 5] shows that the main loss of condensate atoms occurs in the close vicinity of the Feshbach resonance, where all low energy scattering properties become universal [23, 43], i.e. the associated physical observables just depend on the scattering length $a$. The universal parameters $a_{\mathrm{bg}}$ and $\Delta B$ as well as the inverse ramp speed that determine Eq. (33) are also accounted for in the single channel description of the first order microscopic quantum dynamics approach of Ref. [28]. We have, indeed, confirmed that the single channel description also recovers the theoretical loss curves in Fig. 5 as it does in the case of the ${ }^{23} \mathrm{Na}$ Feshbach resonances in Fig. 4 Consequently, our studies clearly reveal that the losses of condensate atoms in linear ramps of the magnetic field strength across Feshbach resonances depend, to a large extent, just on the Landau Zener parameter. The two or multichannel nature of Feshbach resonances is virtually irrelevant to the loss curves in Figs. 4 and 5

In summary, we have shown that physical observables, like the loss of condensate atoms or molecular dissociation spectra, associated with linear upward ramps of a Feshbach resonance level, are largely independent of the microscopic binary collision dynamics and depend just on the parameter $\left|a_{\mathrm{bg}} \| \Delta B\right| /|d B / d t|$ and on the local densities of the atoms in the gas. Consequently, related experimental studies are inconclusive with respect to the details of the interatomic interactions. The interferometric studies in Refs. [3, 4] and their subsequent theoretical analysis in Refs. [26, 28, 32, 39] have demonstrated, however, that the universality of the physical quantities described in this paper is characteristic for linear ramps of the magnetic field strength.

\section{Acknowledgments}

We are particularly grateful to Simon Cornish for providing the experimental data of Ref. [2] to us. We thank Simon Gardiner, Paul Julienne, Keith Burnett, Simon Cornish, and 
Wolfgang Ketterle for many interesting discussions. This research has been supported through a E.C. Marie Curie Fellowship under Contract no. HPMF-CT-2002-02000 (K.G.), a
University Research Fellowship of the Royal Society (T.K.), and by the Deutsche Forschungsgemeinschaft (T.G.).
[1] S. Inouye, M.R. Andrews, J. Stenger, H.-J. Miesner, D.M. Stamper-Kurn, and W. Ketterle, Nature (London) 392, 151 (1998).

[2] S.L. Cornish, N.R. Claussen, J.L. Roberts, E.A. Cornell, and C.E. Wieman, Phys. Rev. Lett. 85, 1795 (2000).

[3] E.A. Donley, N.R. Claussen, S.T. Thompson, and C.E. Wieman, Nature (London) 417, 529 (2002).

[4] N.R. Claussen, S.J.J.M.F. Kokkelmans, S.T. Thompson, E.A. Donley, E. Hodby, and C.E. Wieman, Phys. Rev. A 67, 060701 (2003).

[5] C.A. Regal, C. Ticknor, J.L. Bohn, and D.S. Jin, Nature (London) 424, 47 (2003).

[6] J. Herbig, T. Kraemer, M. Mark, T. Weber, C. Chin, H.C. Nägerl, and R. Grimm, Science 301, 1510 (2003).

[7] S. Dürr, T. Volz, A. Marte, and G. Rempe, Phys. Rev. Lett. 92, 020406 (2004).

[8] K.E. Strecker, G.B. Partridge, and R.G. Hulet, Phys. Rev. Lett. 91, 080406 (2003).

[9] J. Cubizolles, T. Bourdel, S.J.J.M.F. Kokkelmans, G.V. Shlyapnikov, and C. Salomon, Phys. Rev. Lett. 91, 240401 (2003).

[10] S. Jochim, M. Bartenstein, A. Altmeyer, G. Hendl, C. Chin, J. Hecker Denschlag, and R. Grimm, Phys. Rev. Lett. 91, 240402 (2003).

[11] C. A. Regal, M. Greiner, and D. S. Jin, Phys. Rev. Lett. 92, 083201 (2004).

[12] K. Xu, T. Mukaiyama, J.R. Abo-Shaeer, J.K. Chin, D.E. Miller, and W. Ketterle, Phys. Rev. Lett. 91, 210402 (2003).

[13] M. Greiner, C.A. Regal, and D.S. Jin, Nature (London) 426, 537 (2003)

[14] S. Jochim, M. Bartenstein, A. Altmeyer, G. Hendl, S. Riedl, C. Chin, J. Hecker Denschlag, and R. Grimm, Science 302, 2101 (2003).

[15] T. Mukaiyama, J.R. Abo-Shaeer, K. Xu, J.K. Chin, and W. Ketterle, Phys. Rev. Lett. 92, 180402 (2004).

[16] M.W. Zwierlein, C.A. Stan, C.H. Schunck, S.M.F. Raupach, S. Gupta, Z. Hadzibabic, and W. Ketterle, Phys. Rev. Lett. 91, 250401 (2003).

[17] M. Bartenstein, A. Altmeyer, S. Riedl, S. Jochim, C. Chin, J. Hecker Denschlag, and R. Grimm, Phys. Rev. Lett. 92, 120401 (2004).

[18] C. A. Regal, M. Greiner, and D. S. Jin, Phys. Rev. Lett. 92, 040403 (2004).

[19] M. W. Zwierlein, C. A. Stan, C. H. Schunck, S. M. F. Raupach, A. J. Kerman, and W. Ketterle, Phys. Rev. Lett. 92, 120403 (2004).

[20] M. Bartenstein, A. Altmeyer, S. Riedl, S. Jochim, C. Chin, J. Hecker Denschlag, and R. Grimm, Phys. Rev. Lett. 92, 203201 (2004).

[21] J. Stenger, S. Inouye, M.R. Andrews, H.-J. Miesner, D.M. Stamper-Kurn, and W. Ketterle, Phys. Rev. Lett. 82, 2422 (1999).

[22] F.H. Mies, E. Tiesinga, and P.S. Julienne, Phys. Rev. A 61, 022721 (2000).

[23] K. Góral, T. Köhler, S.A. Gardiner, E. Tiesinga, and P.S. Julienne, arXiv.org eprint cond-mat/0312178 (2003).

[24] We note that an upward sweep of the Feshbach resonance level in energy can imply a downward sweep of the magnetic field strength. This is the case when the difference in magnetic moment between the Feshbach resonance level and a pair of atoms in the open channel is negative. This situation occurs, e.g., for the $155 \mathrm{G}$ Feshbach resonance of ${ }^{85} \mathrm{Rb}$ (see Fig. 1).

[25] F.A. van Abeelen and B.J. Verhaar, Phys. Rev. Lett. 83, 1550 (1999).

[26] S.J.J.M.F. Kokkelmans and M.J. Holland, Phys. Rev. Lett. 89, 180401 (2002).

[27] M. Mackie, K.-A. Suominen, and J. Javanainen, Phys. Rev. Lett. 89, 180403 (2002).

[28] T. Köhler, T. Gasenzer, and K. Burnett, Phys. Rev. A 67, 013601 (2003).

[29] P.D. Drummond, K.V. Kheruntsyan, and H. He, Phys. Rev. Lett. 81, 3055 (1998).

[30] E. Timmermans, P. Tommasini, M. Hussein, and A. Kerman, Phys. Rep. 315, 199 (1999).

[31] V.A. Yurovsky, A. Ben-Reuven, P.S. Julienne, and C.J. Williams, Phys. Rev. A 60, 765 (1999).

[32] T. Köhler and K. Burnett, Phys. Rev. A 65, 033601 (2002).

[33] R.A. Duine and H.T.C. Stoof, Phys. Rev. A 68, 013602 (2003).

[34] P. Naidon and F. Masnou-Seeuws, Phys. Rev. A 68, 033612 (2003).

[35] V.A. Yurovsky and A. Ben-Reuven, J. Phys. B 36, 335 (2003).

[36] J.L. Roberts, J.P. Burke, Jr., N.R. Claussen, S.L. Cornish, E.A. Donley, and C.E. Wieman, Phys. Rev. A 64, 024702 (2001).

[37] E.G.M. van Kempen, S.J.J.M.F. Kokkelmans, D.J. Heinzen, and B.J. Verhaar, Phys. Rev. Lett. 88, 093201 (2002).

[38] S. Dürr, T. Volz, and G. Rempe, arXive.org eprint cond-mat/0405606

[39] K. Góral, T. Köhler, and K. Burnett, arXive.org eprint cond-mat/0407627

[40] T. Köhler, Phys. Rev. Lett. 89, 210404 (2002).

[41] Within the range of ramp speeds of Ref. [2] in which the remnant condensate was nearly completely depleted analogous measurements on a thermal ${ }^{85} \mathrm{Rb}$ gas $(T=430 \mathrm{nK})$, with a comparable mean density, did not exhibit any significant losses [2]. Consequently, we expect inelastic collisions to be negligible because these losses should otherwise be visible also in the thermal gas.

[42] G.F. Gribakin and V.V. Flambaum, Phys. Rev. 48, 546 (1993).

[43] T. Köhler, T. Gasenzer, P.S. Julienne, and K. Burnett, Phys. Rev. Lett. 91, 230401 (2003).

[44] P. Kharchenko, J.F. Babb, and A. Dalgarno, Phys. Rev. A 55, 3566-3572 (1997).

[45] Paul Julienne, private communication.

[46] P.S. Julienne, E. Tiesinga, and T. Köhler, J. Mod. Opt. 51, 1787 (2004).

[47] We note that the elimination of the pair function conveniently reduces the spatial degrees of freedom from six dimensions in Eqs. (2) and (3) to three dimensions in Eq. (16).

[48] We note that $|\mathrm{cl}\rangle \phi_{\text {res }}(r)$ is not a bound state of the two-body Hamiltonian (4). This state would thus dissociate.

[49] S.L. Cornish, private communication.

[50] W. Ketterle, private communication. 
[51] For the $853 \mathrm{G}$ resonance of ${ }^{23} \mathrm{Na}$ we have used the theoretical value of $\Delta B=0.01 \mathrm{G}$ [21] for the resonance width, which is a factor of 4 larger than the experimental width reported in Ref. [21].
[52] Yu.N. Demkov and V.I. Osherov, Sov. Phys. JETP 26, 916 (1968). 